\section{Gold nanoparticles}

designed for

combining dual

modality imaging

and radiotherapy

\author{
Christophe Alric ${ }^{1}$, Raphaël Serduc ${ }^{2}$, \\ Céline Mandon ${ }^{3}$, Jacqueline Taleb ${ }^{3}$, \\ Géraldine Le Duc ${ }^{2}$, Alice Le Meur-Herland ${ }^{3}$, \\ Claire Billotey ${ }^{3}$, Pascal Perriat ${ }^{4}$, \\ Stéphane Roux ${ }^{1}$, Olivier Tillement ${ }^{1}$
}

\author{
${ }^{1}$ Laboratoire de Physico-Chimie des Matériaux \\ Luminescents, UMR 5620 CNRS - Université Claude \\ Bernard Lyon 1, 69622 Villeurbanne Cedex, France \\ 2 European Synchrotron Radiation Facility, ID 17 \\ biomedical Beamline, Polygone Scientifique Louis \\ Néel, 6 rue Jules Horowitz, 38000 Grenoble, France \\ ${ }^{3}$ Laboratoire CREATIS-LRMN - Animage, UMR 5220 \\ CNRS - U630 INSERM - INSA de Lyon - Université \\ Claude Bernard Lyon 1, 69622 Villeurbanne \\ Cedex, France \\ ${ }^{4}$ Matériaux Ingénierie et Science, UMR 5510 CNRS \\ - INSA de Lyon, 69621 Villeurbanne Cedex, France \\ E-mail: roux@pcml.univ-lyon1.fr
}

\begin{abstract}
The synthesis of gold nanoparticles functionalized by gadolinium chelates constitutes an attractive way for combining imaging and therapy. The presence of gadolinium chelates allows monitoring their biodistribution after intravenous injection in small animals by magnetic resonance imaging (MRI) while the gold core strongly absorbs the $\mathrm{X}$-ray photons. This feature is exploited for X-ray imaging but also for radiotherapy.
\end{abstract}

\section{Introduction}

One of the most promising applications of nanosized gold objects lies in their ability to combine imaging and therapy. Two main strategies were developed for designing gold particles that can be used for imaging and destroying tumors. The first one results from the properties of gold element whereas the second one rests on the tuneable optical properties of gold particles. Gold particles are indeed characterized by strong light diffusion and absorption cross sections which are sensitive to the size, the shape and the dielectric environment [1,2]. Thus, these particles act as novel contrast agents for optical detection due to their enhanced scattering. Moreover their use for photothermal therapy can be envisaged since the strongly absorbed radiation is efficiently converted into heat. For instance, Drezek et al. demonstrated that silica cores (diameter: $120 \mathrm{~nm}$ ) embedded in a thin gold layer (thickness: $10 \mathrm{~nm}$ ) behaves both as contrast and therapeutic agents when NIR radiation is used [3]. Similar behavior was observed by El-Sayed et al. with gold nanorods [4]. Due to the weak depth penetration of NIR light, the application of these particles for in vivo imaging and therapy of cancer is restricted to superficial treatment. Despite these convincing proof-of-concept experiments, the biomedical applications of these particles are likely to be impeded by their large size since it was demonstrated that the free circulation without undesirable non-specific accumulation and the renal elimination of the particles which are prerequisites for the delivery of contrast agents and drugs by intravenous injection is observed only for ultrasmall particles (hydrodynamic diameter $<5 \mathrm{~nm}$ ) [5]. Unfortunately the reduction of the size of these objects does not allow overcoming this hurdle because it is accompanied by a blue shift of the radiation which is used for inducing the photothermal effect. This is detrimental for biomedical applications because the penetration depth of short wavelength of visible light is very weak.

The second strategy explored for combining imaging and therapy with gold particles is based on the strong X-ray absorption cross section due to the high $\mathbf{Z}$ of gold element. The work done by Hainfeld et al. showed that $1.9 \mathrm{~nm}$ sized gold nanoparticles can be followed up with X-ray imaging techniques in blood pool after intravenous injection to mice with a tumor in legs $[6,7]$. Due to a higher vascularization than the surrounding tissue, the amount of contrast agents was therefore more important in the tumor which can be more clearly delineated. Pharmacokinetic studies confirmed that gold nanoparticles preferentially located in the tumor since the tumor-to-normal tissue gold ratio was about 8:1. This preferential localization is a key parameter for significant high-Z radioenhancement since the survival of tumor bearing mice was greatly more lengthened when they were treated both with gold nanoparticles and X-ray radiation. The preferential delivery of high content of gold nanoparticles to the tumor improves the X-ray therapy owing to the 
<smiles>O=C(O)CN(CCN(CC(=O)O)CC(=O)O)CCN(CC(=O)O)CC(=O)O</smiles>

DTPA

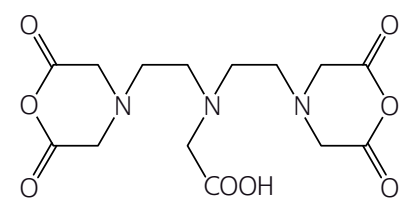

DTPA-DA

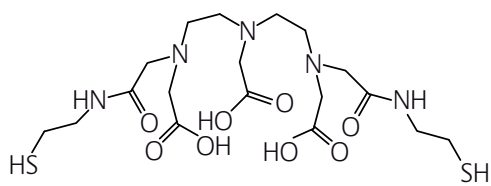

DTDTPA<smiles>O=C(O)CN(CCN1CC(=O)OC(=O)C1)CCN1CC(=O)OC(=O)C1</smiles>

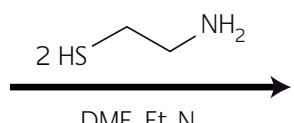

DMF, $\mathrm{Et}_{3} \mathrm{~N}$

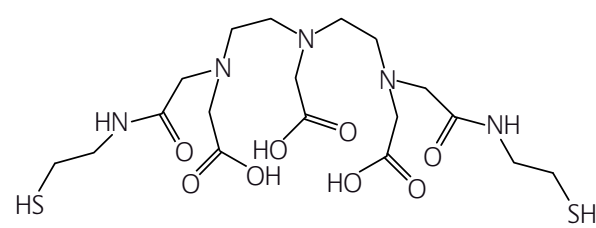

(b)

(a)

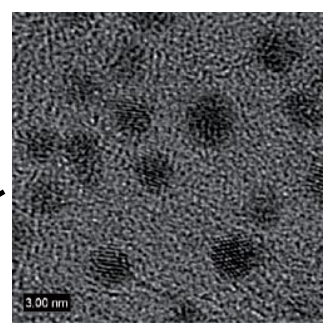

$\varnothing=2.4 \mathrm{~nm} \pm 0.5 \mathrm{~nm}$

Figure 1

Chemical formulae of DTPA, DTPA-DA and DTDTPA. Synthesis of DTDTPA and of Au@DTDTPA. (a) Colloidal solution of Au@DTDTPA after purification $\left([A u]=50.7 \mathrm{mM}\right.$, i.e. $\left.10 \mathrm{~g} \mathrm{Au} \mathrm{L}^{-1}\right)$. (b) Transmission electron micrograph of Au@DTDTPA nanoparticles

radioenhancement assigned to gold element. This pioneering work has opened the door to the biomedical application of ultrasmall gold nanoparticles since they combine in vivo $\mathrm{X}$-ray imaging with $\mathrm{X}$-ray therapy. Before they are applied in clinical protocol to human patients, these particles constitute an excellent tool for monitoring therapeutic protocol in small animals from the injection to the renal elimination via the ablation of the tumor. However X-ray imaging is not completely harmless for envisaging a long-term biodistribution study without interference with radiotherapy. Moreover this technique suffers of a low sensitivity.

In order to circumvent this problem, the use of gold nanoparticles designed for magnetic resonance imaging was proposed [8,9]. Magnetic resonance imaging (MRI) is a powerful non-invasive technique for establishing in real time a 3D cartography of living bodies with a high resolution. The contrast of the resulting images can be enhanced by the injection of paramagnetic or superparamagnetic agents [10]. Paramagnetic agents ( $\mathrm{Gd}^{3+}$ containing compounds) induce a positive contrast (i.e. their presence is detected by bright zones) whereas a negative contrast (i.e. darkening) occurs when superparamagnetic compounds, which are essentially iron oxide nanocrystals, are used. We previously demonstrated that ultrasmall gold nanoparticles (diameter $2.4 \mathrm{~nm}$ ) can enhance the contrast of MRI images if they are functionalized by gadolinium chelates $[8,9]$.

\section{Experimental section}

\section{Chemicals}

Reagents were purchased from Aldrich. Organic solvents (reagent grade) were purchased from SDS and used as received. For the preparation of an aqueous solution and for the rinsing of gold nanoparticles, only milli-Q water ( $\rho>18 \mathrm{M} \Omega$ ) was used. The filtration of gold nanoparticles was performed on polymer membrane of $0.22 \mu \mathrm{m}$ pore diameter purchased from Osmonics Inc.

\section{Functionalized gold nanoparticles preparation}

The synthesis, described by Brust et al., consists in reducing $\mathrm{HAuCl}_{4}, 3 \mathrm{H}_{2} \mathrm{O}$ by $\mathrm{NaBH}_{4}$ in presence of thiols (stabilizers) which, by adsorption on growing particles, ensure the control of the size and the stability of the colloid [11]. The synthesis of gold nanoparticles coated by gadolinium chelates requires the use of molecules which are able both to strongly interact with the surface of gold nanoparticles and to coordinate paramagnetic gadolinium $\left(\mathrm{Gd}^{3+}\right)$ ions. Since diethylenetriaminepentaacetic acid (DTPA) is an efficient ligand for the preparation of gadolinium chelates (DTPA-Gd), a dithiolated derivative of DTPA (DTDTPA) was synthesized from diethylenetriaminepentaacetic acid dianhydride (DTPA-DA) (Figure 1) [8].

For a typical preparation of gold particles, $200 \mathrm{mg}\left(51 \times 10^{-5}\right.$ mol) of $\mathrm{HAuCl}_{4}, 3 \mathrm{H}_{2} \mathrm{O}$, dissolved in $60 \mathrm{~mL}$ methanol were 
placed in a $250 \mathrm{~mL}$ round bottom flask. $256 \mathrm{mg}\left(50 \times 10^{-5}\right.$ mol) of DTDTPA in $40 \mathrm{~mL}$ of water and $2 \mathrm{~mL}$ of acetic acid were added to the gold salt solution under stirring. The mixture turned from yellow to orange. After 5 minutes, $185 \mathrm{mg}\left(489 \times 10^{-5} \mathrm{~mol}\right)$ of $\mathrm{NaBH}_{4}$ dissolved in $14 \mathrm{~mL}$ water were added to the orange mixture under vigorous stirring at room temperature. At the beginning of the $\mathrm{NaBH}_{4}$ addition, the solution became first dark brown then a black floculate appeared.

The vigorous stirring was maintained for $1 \mathrm{~h}$ before adding 5 $\mathrm{mL}$ of $1 \mathrm{M}$ aqueous hydrochloric acid solution. After the partial removal of the solvent under reduced pressure and at maximum $40^{\circ} \mathrm{C}$, the precipitate was filtered on a polymer membrane, washed thoroughly and successively with $0.01 \mathrm{~N} \mathrm{HCl}$, water and diethyl ether. The resulting black powder (Au@DTDTPA) was dried and stocked in the solid state or dispersed in $10 \mathrm{~mL}$ of $0.01 \mathrm{M} \mathrm{NaOH}$ solution (up to $300 \mathrm{mg}$ of dry powder).

\section{Complexation of $\mathrm{Gd}^{3+}$ with Au@DTDTPA}

$\mathrm{Gd}^{3+}$ complexation is carried out through the addition of $\mathrm{GdCl}_{3}$ on Au@DTDTPA colloidal solution under stirring at room temperature. The quantity of $\mathrm{Gd}^{3+}$ is monitored by colorimetric titration with xylenol orange.

\section{Cell line culture and labeling}

The human cervix carcinoma HeLa cell line were grown in DMEM (Dulbecco's Modified Eagle's Medium) from Invitrogen/ GibcoBRL (Cergy Pontoise, France) supplemented with 10\% foetal calf serum (FCS), from Dutscher (Brumath, France), 1 $\mathrm{mg} / \mathrm{ml}$ Fungizone and $50 \mathrm{u} / \mathrm{ml}$ Penicillin / Streptomycin, in standard cell culture conditions $\left(37^{\circ} \mathrm{C}, 5 \% \mathrm{CO}_{2}, 95 \%\right.$ humidity).

After 2 days of cell culture, HeLa cells, seeded with a cell suspension concentration of $7.5 \times 10^{4}$ cells $/ \mathrm{ml}$ in Petri dishes, were incubated for 1 hour within the buffered solution of incubation containing Au@DTDTPA or Au@DTDTPA-Gd . $_{50}$. Negative control was represented by buffered sucrose solution containing $250 \mathrm{mM}$ sucrose.

\section{Splenocytes extraction and labeling}

Spleen lymphocytes were isolated from fresh spleen obtained from euthanasiated mice. Briefly, spleens were mechanically homogenized in RPMI medium, filtered (30 mm nylon mesh) and washed in RPMI. Red cells were depleted by incubating the spleen cell suspension for 5 min at room temperature, with $\mathrm{NH}_{4} \mathrm{Cl}-\mathrm{HCl}$ lysis buffer, for $5 \mathrm{~min}$ at room temperature.

Ice-cold RPMI was added immediately after lysis time to the cell suspensions. Cells were pelleted (1100 rpm; $4^{\circ} \mathrm{C} ; 5$ min) and the cell pellets were resuspended, washed and centrifuged twice in ice-cold RPMI. The final pellets were resuspended in buffered solution of incubation as previously described.

\section{Cellular viability measurement}

Splenocytes and HeLa cells viability was evaluated through the trypan blue dye exclusion method. This vital stain is used to selectively color dead cells blue (and are shown as a distinctive blue color under a microscope), since in live cells, with intact cell membranes, Trypan blue is not absorbed. Cells were counted in Malassez counting cell using an inverted fluorescent microscope.

\section{Anesthesia}

The male Fisher 344 rats (180-280 g at arrival, Charles River, L'Arbresle, France) were anesthetized with a first 4\% isoflurane inhalation shot followed by an intraperitoneal injection of a mixture of ketamine $(64.5 \mathrm{mg} / \mathrm{kg})$ and xylazine $(5.4 \mathrm{mg} / \mathrm{kg})$, twice (implantation, irradiation).

\section{Intracerebral models in rat brain}

The $9 \mathrm{~L}$ gliosarcoma cell line [12] was established by Benda et al. [13]. Cells were grown with complete medium at $37^{\circ} \mathrm{C}$. After anesthesia, the rats were placed on a stereotactic head holder (model 900, David Kopf Instruments, Tujunga, USA). At DO, $10^{4}$ cells were suspended into $1 \mu \mathrm{l}$ DMEM with antibiotics (1\%) then injected using a $1 \mu \mathrm{L}$ Hamilton syringe through a burr hole in the right caudate nucleus ( $9 \mathrm{~mm}$ anterior to the ear-bars i.e. at bregma site, 3.5 $\mathrm{mm}$ lateral to the midline, $5.5 \mathrm{~mm}$ depth from the skull) $[14,15]$. All delays expressed in the manuscript are postimplantation. All operative procedures related to animal care strictly conformed to the Guidelines of the French Government with licenses 380324/380456 and A3818510002 and were reviewed by the Comité d’Ethique Régional Rhône-Alpes.

\section{$T_{1}$ measurements and MR imaging}

$\mathrm{T}_{1}$ measurements and MR imaging were performed at $7 \mathrm{~T}$ using an inversion recovery FLASH (IR-FLASH) imaging sequence with varying IR time (Biospec System 70/20, Brucker, Ettlingen, Germany). $\mathrm{T}_{1}$-weighted contrast enhancement was controlled running a standard Spin-Echo (SE) sequence with $500 \mathrm{~ms}$ TR and $12 \mathrm{~ms}$ TE. For in vivo imaging, anaesthesia was induced with 1.8-2\% isoflurane and maintained with $1.4-1.6 \%$ isoflurane in a mixture of $\mathrm{O}_{2} / \mathrm{N}_{2}(25 / 75 \%)$.

\section{Synchrotron Radiation Computed Tomography (SRCT) imaging}

SRCT images of phantoms containing Au@DTDTPA at various gold concentrations and in vivo imaging were performed via the biomedical beamline ID17 from the European Synchrotron Radiation Facility (Grenoble, France). The SRCT images were obtained by positioning the center of the sample in the convergence point of two beams bracketing the gold $\mathrm{K}$ edge energy $(80.7 \mathrm{keV})$. After they crossed at the center of the sample (they can be considered as superimposed over the whole sample width), these beams diverged to match two distinct lines on the detector, allowing the simultaneous acquisition of attenuation profiles at $80.5 \mathrm{keV}$ and $80.9 \mathrm{keV}$ (below and above the gold K-edge, respectively). From the logarithmic subtraction of the attenuation profiles acquired simultaneously at two energies above and below the gold 


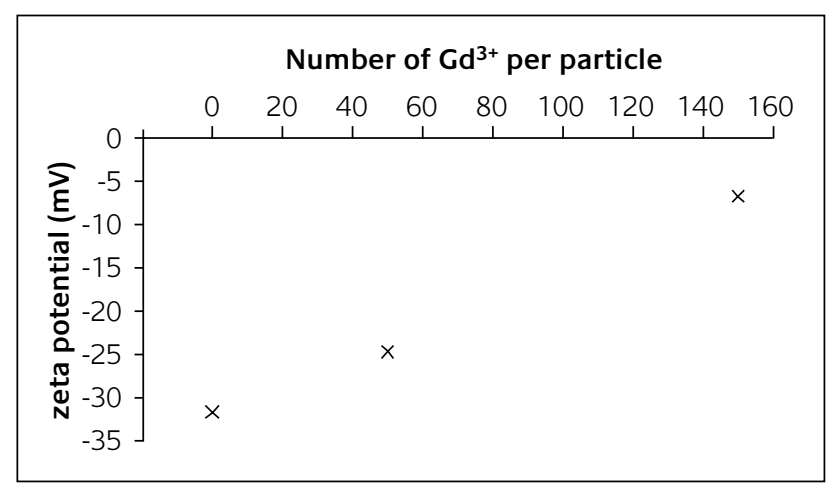

Figure 2

Evolution of the $\xi$-potential of Au@DTDTPA-Gd versus the number of Gd ${ }^{3+}$ per particle

K-edge, accurate absolute gold concentrations can be determined. This method has been described elsewhere $[16,17]$. The injections and the imaging experiments were carried out on rats which were anesthetized by intraperitoneal injection of a mixture of xylazine/ketamine.

\section{Synchrotron based Microbeam Radiation Therapy (MRT)}

At the ESRF, one beamline has been dedicated to biomedical research and is described elsewhere [16]. The X-ray flux delivered by the ESRF storage ring exceeds the flux of a tungsten-anode $x$-ray tube by 5 orders of magnitude.

The present MRT study uses a white beam spectrum with its maximum intensity at $83 \mathrm{keV}$ (mean energy $=100 \mathrm{keV}$, critical energy $=33 \mathrm{keV}$ ) filtered by Be: $0.5 \mathrm{~mm}$, vitreous carbon: $1.42 \mathrm{~mm}, \mathrm{Al}: 1.52 \mathrm{~mm}, \mathrm{Cu}: 1.04 \mathrm{~mm}$ (to avoid the presence of low penetrating $X$-rays). Fourteen days after the tumor implantation, the rats were placed perpendicularly to the beam (for unidirectional irradiation and for the first irradiation of the cross firing geometry), and on the beam track (in antero-posterior mode for the other direction in cross firing mode). The skin entrance dose delivered was $\sim 460$ Gy in a single exposure. The field of irradiation was $11 \mathrm{~mm}$ high and $10 \mathrm{~mm}$ wide, centered on the tumor, with a $1 \mathrm{~mm}$ overlap with air at the top of the skull. It contained 51 microbeams characterized by an average width of $25 \mu \mathrm{m}$ and a center to center spacing of microbeams equal to $200 \mu \mathrm{m}$ as described earlier [18]. The rats were irradiated in cross fired mode first in lateral position from right to left, second in antero-posterior position.

\section{Table 1}

Survival results for non treated rats (control group) and for treated rats (irradiation 20 minutes after the intravenous injection of Au@DTDTPA$G d_{50}\left(n=8, V=1.4 \mathrm{~mL},[\mathrm{Au}]=50.7 \mathrm{mM}\right.$, i.e. $\left.\left.10 \mathrm{~g} \mathrm{Au} L^{-1},[G d]=5.00 \mathrm{mM}\right)\right)$

\begin{tabular}{lccc}
\hline Series & $\mathbf{n}$ & MST days & MeST days \\
\hline $\begin{array}{l}\text { Control group } \\
\text { Au@DTDTPA-Cd }\end{array}$ & 6 & $17.66 \pm 0.33$ & 17.5 \\
+ irradiation & 8 & $33.25 \pm 11.75$ & 27.5 \\
\hline
\end{tabular}

\section{Experimental groups}

As plotted in Table 1, 6 rats were used as non irradiated controls. Other rats received Au@DTDTPA-Gd delivered to the rats $(n=8)$ by intravenous injection $(1.4 \mathrm{~mL},[\mathrm{Au}]=50.7$ $\mathrm{mM}$ and $[\mathrm{Gd}]=5 \mathrm{mM}$ ) in a tail vein and were exposed to the microbeam radiation (vide supra).

\section{Survival analysis and follow up}

The clinical status of the rats was checked 3 times per week. The relative average body weight (RAW, i.e. average at day D minus average weight at D0) of each rat was plotted versus time. At later tumor stage, rats were euthanized by intracardiac injection of pentobarbital sodium (150 mg. $\mathrm{kg}^{-1}$ ) less than 1 day before their anticipated death as judged by clinical signs. Some of them were found dead. The time between implantation and death was recorded as survival time (one day was added for euthanized rats). The mean survival time (MST) and the median survival time (MeST) were calculated for each group and Kaplan Meier survival data were plotted versus time after tumor implantation.

\section{Results and discussion}

These particles were synthesized by reducing the gold salt with $\mathrm{NaBH}_{4}$ in presence of a dithiolated derivative of DTPA (DTDTPA) (Figure 1). The resulting particles are easily dispersed in aqueous solution (Figure 1a) up to $15 \mathrm{~g} \mathrm{Au} \mathrm{L}^{-1}$ and have an average size of $2.4 \mathrm{~nm}$ (Figure 1b). In addition to control the particle growth, DTDTPA molecules established a network strongly anchored on the gold nanoparticles (Au@DTDTPA) since it was previously demonstrated that a part of DTDTPA is directly tethered to the gold surface thanks to one or two thiol ends. The second thiol group allows the coupling with DTDTPA molecules which were not directly anchored to the gold surface via disulfide bounds. As a result, gold core is embedded in a multilayered shell of DTDTPA ligands [8]. The presence of DTDTPA network on the particles confers a high colloidal stability in biological media and allows the immobilization of $\mathrm{Gd}^{3+}$ ions (until 150 per particle) in the organic shell. However the immobilization of $\mathrm{Cd}^{3+}$ ions in the negatively charged DTDTPA shell induces a decrease of $\xi$-potential which is detrimental for the colloidal stability (Figure 2). When there are $150 \mathrm{Gd}^{3+}$ ions per particle (Au@DTDTPA-Gd ${ }_{150}$ ), colloidal stability is only observed for gold content smaller than $1 \mathrm{~g} . \mathrm{L}^{-1}$. The negative charge of Au@DTDTPA-Gd ${ }_{150}$ is indeed not sufficiently high ( $\xi$-potential $=-7 \mathrm{mV}$ ) for avoiding the agglomeration of the particles. The presence of $50 \mathrm{Gd}^{3+}$ ions in the organic shell of each nanoparticle (Au@DTDTPA-Gd ${ }_{50}$ ) constitutes a good compromise since $\xi$-potential is sufficiently high $(-25 \mathrm{mV}$ ) for ensuring colloidal stability over several weeks for gold content higher than 10 g.L.-1. Moreover Au@DTDTPA-Gd ${ }_{50}$ colloid with a gold content of $10 \mathrm{~g} . \mathrm{L}-1$ contains more $\mathrm{Gd}^{3+}$ ions ( $5 \mathrm{mM}$ ) than Au@DTDTPA-Gd ${ }_{150}$ colloid whose stability requires that gold content does not exceed 1 g. $\mathrm{L}^{-1}$ (i.e. $\left[\mathrm{Gd}^{3+}\right]<2 \mathrm{mM}$ ). 


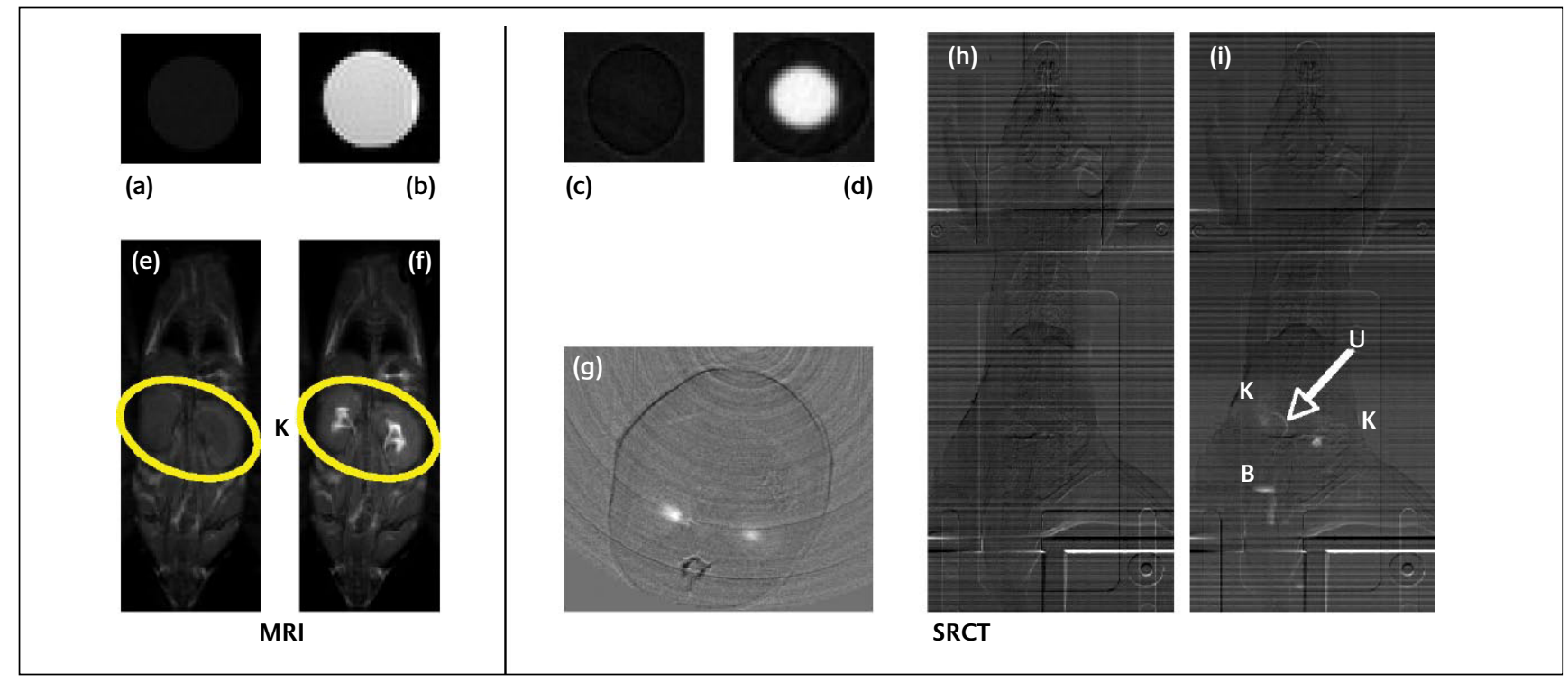

Figure 3

$T_{1}$-weighted magnetic resonance images (a) of water as negative control, (b) of aqueous colloid of Au@DTDTPA-Gd . $_{\text {. }}$. SRCT images of phantoms containing (c) water and (d) Au@DTDTPA nanoparticles. T,-weighted magnetic resonance images of a mouse (e) before and (f) 45 minutes after intravenous injection of Au@DTDTPA-Gd ${ }_{50}$ (g) SRCT images of transverse slice including kidneys recorded 6 minutes after injection of Au@DTDTPA-Gd ${ }_{50}$ to a rat (slice thickness: $1 \mathrm{~mm}$ ). Planar X-ray images in transmission mode of a rat (h) before and (i) 10 minutes after intravenous injection of Au@DTDTPA-Gd ${ }_{50}\left(K_{\text {for }}\right.$ kidneys, $U$ for ureter and $B$ for bladder). For b, f, $g$ and $i:[A u]=50.7 \mathrm{mM}$, i.e. $10 \mathrm{~g} \mathrm{Au} L^{-1},[G d]=5.00 \mathrm{mM}$. For d: $[\mathrm{Au}]=50.7 \mathrm{mM}$

Since they ally a high gold and gadolinium content and colloidal stability over several weeks, Au@DTDTPA-Gd colloids appear therefore very attractive for MRI and X-ray applications. Due to $\mathrm{Gd}^{3+}$ ions entrapped in the DTDTPA shell, these particles (Au@DTDTPA-Gd ${ }_{50},[\mathrm{Au}]=50.7 \mathrm{mM}$, i.e. $10 \mathrm{~g}$ $\mathrm{Au} \mathrm{L}^{-1}$ and $[\mathrm{Gd}]=5 \mathrm{mM}$ i.e. $50 \mathrm{Gd}^{3+}$ per particle) induce a strong contrast enhancement of MRI images (Figures 3a-3b). The phantom containing Au@DTDTPA-Gd $\mathrm{d}_{50}$ nanoparticles (Figure $3 b$ ) is brighter than the negative control which is composed of only water (Figure 3a). As expected, they are also detected by X-ray SRCT. Figures 3c-3d obviously show that the presence of gold nanoparticles is revealed by a strong enhancement of the contrast of the SRCT image. The biocompatibility of Au@DTDTPA and Au@DTDTPA-Gd was checked by mixing gold nanoparticles with splenocytes and HeLa cells (Figure 4). The viability of cells (splenocytes and HeLa) incubated for 1 hour in buffered sucrose solution (250 mM) containing Au@DTDTPA ([Au] = 2 and 4 mM) or Au@ DTDTPA-Gd $_{50}([\mathrm{Au}]=4 \mathrm{mM})$ was determined by trypan blue exclusion and compared to the one of cells incubated for 1 hour in buffered sucrose solution (250 mM) but without nanoparticles (negative control). Figure $4 a$ shows that the proportion of cell death is small when splenocytes are incubated in presence of nanoparticles ([Au] $=4 \mathrm{mM}$ ) and is of same range than cells incubated without nanoparticle. Similar results are obtained after incubation of HeLa cells in

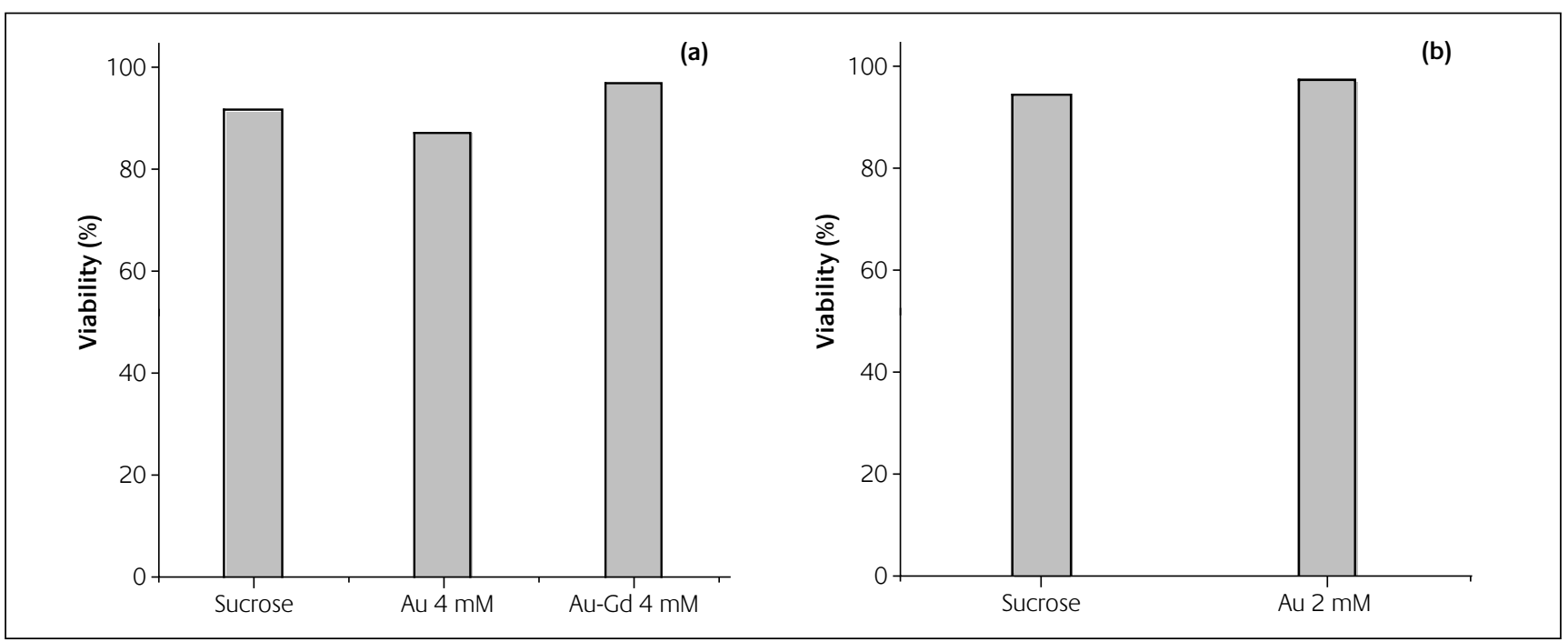

Figure 4

Cytotoxicity of Au@DTDTPA (Au) and Au@DTDTPA-Gd 50 (Au-Gd) on (a) splenocytes and (b) HeLa cells. Sucrose: buffered solution of incubation containing 250 mM sucrose (negative control); Au 2 mM and Au 4 mM: buffered solution of incubation containing 250 mM sucrose and Au@DTDTPA nanoparticles ([Au]=2 and 4 mM, respectively); Au-Gd 4 mM: buffered solution of incubation containing 250 mM sucrose and Au@DTDTPA-Gd ${ }_{50}$ nanoparticles ([Au]=4 mM) 


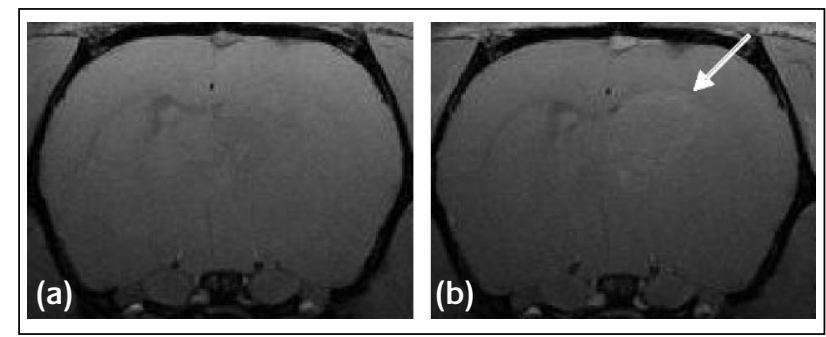

\section{Figure 5}

$T_{1}$-weighted image of brain of tumor-bearing rat (a) before and (b) 20 minutes after intravenous injection of Au@DTDTPA-Gd

sucrose solution containing Au@DTDTPA ([Au]=2mM, Figure 4b). From these data, we can deduce that the cytoxicity of Au@DTDTPA and Au@DTDTPA-Gd ${ }_{50}([\mathrm{Au}]=4 \mathrm{mM})$ is weak. The innocuousness of these particles was confirmed by in vivo experiments. The intravenous injection of Au@DTDTPA-Cd $\left([\mathrm{Au}]=50.7 \mathrm{mM}\right.$, i.e. $10 \mathrm{~g} \mathrm{Au} \mathrm{L}^{-1}$ and $[\mathrm{Gd}]=5 \mathrm{mM}$ i.e. $50 \mathrm{Gd}^{3+}$ per particle) is well tolerated by small animals (mice, rats) and no overt clinical sign was observed for at least two months after the injection. Since they are able to induce a contrast enhancement of MRI and X-ray images, these particles can be easily followed up. The presence of Au@DTDTPA-Cd $\mathrm{d}_{50}$ is revealed by the appearance of bright zone after their injection (Figures 3e-3i). The examination of images acquired by MRI and $\mathrm{X}$-ray imaging experiments revealed that bright zones were only observed in kidneys, ureters and bladder. This indicates that the particles freely circulate in the blood pool without undesirable non-specific accumulation (as confirmed by ICP-MS analysis of the organs) and are cleared from the body through the kidneys when they are injected to healthy rats (Figures 3e-3i) [9]. In contrast to the case of healthy animals, Au@DTDTPA-Gd ${ }_{50}$ nanoparticles crossed the brain blood barrier (BBB) of rats bearing a tumor in brain (9L gliosarcoma). A moderate contrast enhancement (15\%) was observed in the tumor zone until 20 minutes after the injection (Figure 5). This indicates that gold nanoparticles are able to reach this region in the case of unhealthy rats. Indeed the presence of the tumor

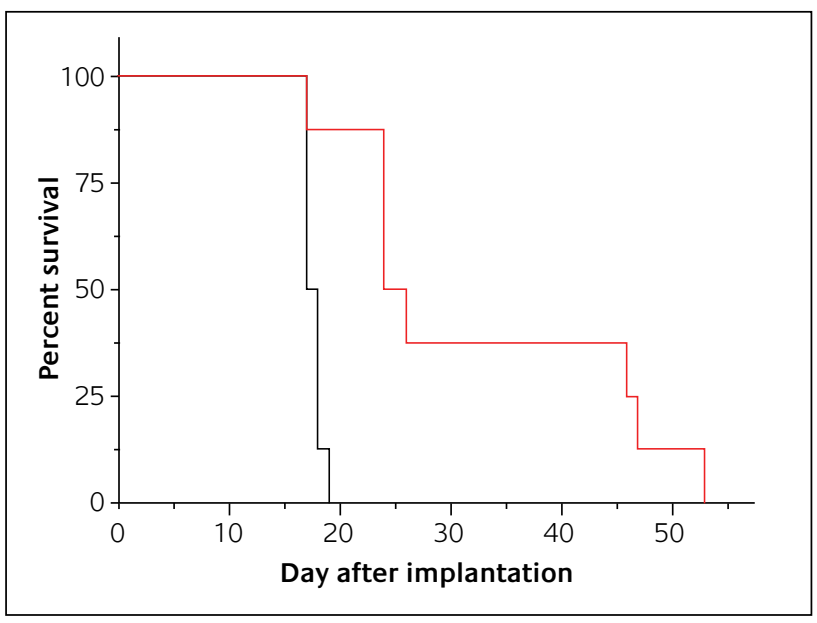

\section{Figure 6}

Graph of rats survival. Black curve: non treated rats (control group, $n=6)$. Red curve: irradiation of rats 20 minutes after the injection of Au@ DTDTPA-Gd $d_{50}\left(n=8, V=1.4 \mathrm{~mL},[\mathrm{Au}]=50.7 \mathrm{mM}\right.$, i.e. $10 \mathrm{~g} \mathrm{Au} \mathrm{L}^{-1},[\mathrm{Gd}]=$ $5.00 \mathrm{mM})$ in brain renders BBB leaky and facilitates therefore the crossing of the particles. Moreover the difference of contrast between tumor and surrounding healthy tissue can be explained by a denser vascularisation of the tumor since its rapid growth requires the recruitment of additional blood vessels (angiogenesis) for delivering a large amount of nutriments and oxygen to the tumor $[19,20]$. The preferential accumulation of Au@DTDTPA-Gd nanoparticles was exploited for treating the tumor with $X$-ray beam. The irradiation of tumor bearing rats with X-ray microbeam 20 minutes after Au@DTDTPA-Gd injection led to a longer survival of the rats (Figure 6 and Table 1). As compared with the median survival time (MeST) and mean survival time (MST) of non-treated rats, the rats which received gold nanoparticles and were submitted to X-ray beam exhibit higher MeST (27.5 days versus 17.5) and MST (33.25 \pm 11.75 days versus $17.66 \pm 0.33$ days).

These preliminary experiments are encouraging but improvements are required since a preferential localization is rarely sufficient for sparing the surrounding healthy tissue. The next step of our work will consist in the functionalization of Au@DTDTPA-Gd by peptide for an active targeting of the growing neo-vasculature which supplies nutriment and oxygen to tumors. The destruction of this neo-vasculature is thought to play a major role in the eradication of some vascularized tumors because the resulting deprivation of life-sustaining nutrients and oxygen will prevent the tumor growth [21]. In addition to their ability to immobilize $\mathrm{Gd}^{3+}$ ions and to ensure a high colloidal stability, the DTDTPA shell can also act as anchorage site for organic molecules or biomolecules. Each DTDTPA moiety in the organic shell carries three carboxylic acid groups which can be used for coupling aminated molecules via the formation of an amide linkage. Poly(ethylene glycol) whose both extremities ends by amine $\left(\mathrm{PEG}\left(\mathrm{NH}_{2}\right)_{2}\right)$ was used for demonstrating the possibility to perform a post-functionalization of Au@DTDTPA nanoparticles. Before adding $\mathrm{PEG}\left(\mathrm{NH}_{2}\right)_{2}$ to the gold nanoparticles, $\mathrm{COOH}$ groups were converted to $\mathrm{N}$-hydroxysuccinimidyl ester in presence of EDC (a water soluble carbodiimide) in order to increase the yield of PEG coupling in water. If the surface of Au@DTDTPA nanoparticles is derivatized by $\mathrm{PEG}\left(\mathrm{NH}_{2}\right)_{2}$, the anchorage of these particles is expected onto carboxylated silica particles via the formation of amide bond between the intact amino group of the polymer tethered onto the gold nanoparticles and the carboxylic acid groups of the silica particles which were converted to N-hydroxysuccinimidyl ester. Figure 7a shows that gold nanoparticles, which were in contact with PEG $\left(\mathrm{NH}_{2}\right)_{2}$, are bound to the carboxylated silica nanoparticles whereas unmodified Au@DTDTPA nanoparticles are not anchored onto the silica nanoparticles (Figure 7b). This constitutes an evidence of the presence of PEG $\left(\mathrm{NH}_{2}\right)_{2}$ onto Au@DTDTPA particles. This experiment demonstrates that Au@DTDTPA nanoparticles can be functionalize by aminated molecules in water and paves therefore the way to the targeted imaging and therapy since the functionalization of Au@DTDTPA by bio-targeting groups can be performed. 


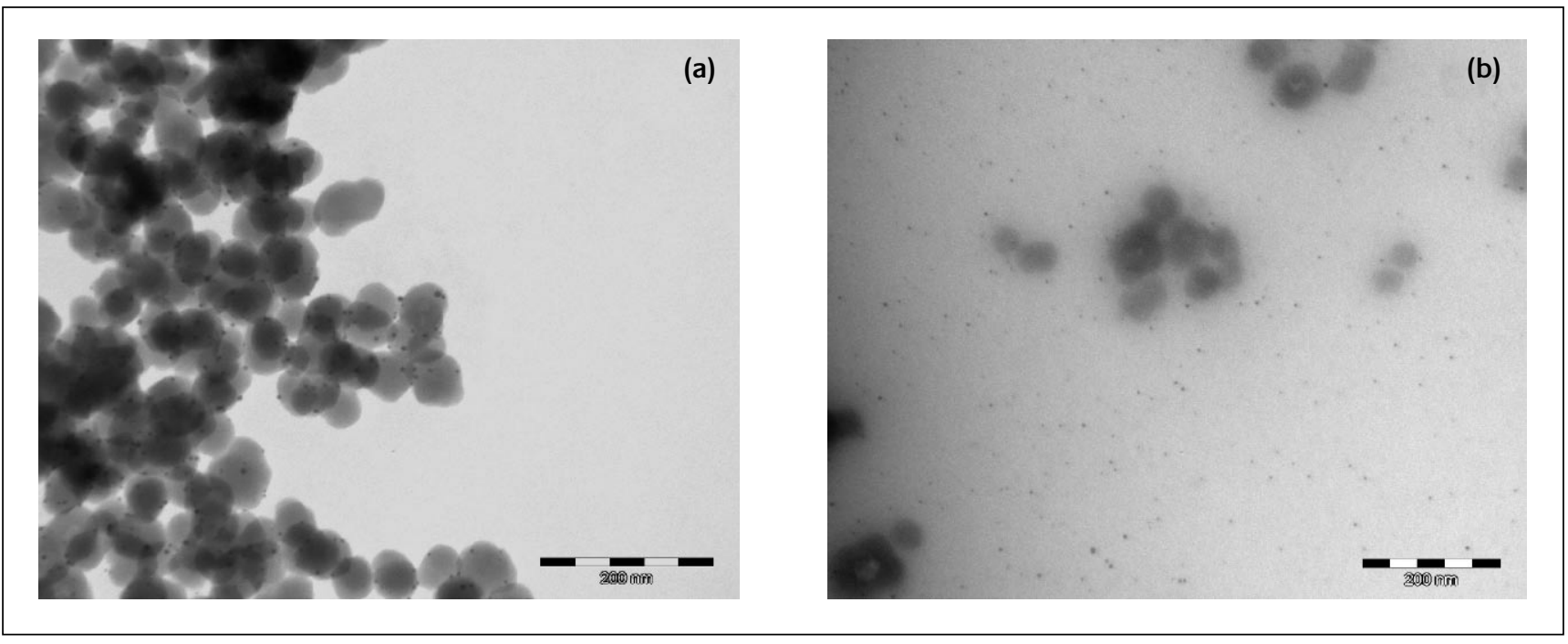

Figure 7

TEM micrographs of (a) Au@DTDTPA-PEG-NH ${ }_{2}$ onto carboxylated silica nanoparticles and (b) a mixture of Au@DTDTPA and carboxylated silica nanoparticles. The smallest black dots are gold nanoparticles

\section{Conclusion}

Owing to their peculiar design, Au@DTDTPA-Gd nanoparticles appear very well suited for combining in vivo imaging and radiotherapy. The presence of gold element and gadolinium ions in Au@DTDTPA-Gd confers to the particles the ability to improve the survival of rats bearing a very aggressive tumor and to follow up them both by X-ray imaging and MRI. Moreover DTDTPA molecules which allow the immobilization of $\mathrm{Gd}^{3+}$ ions on the particles and favor the water solubility of these particles in biological media afford also the opportunity of further functionalization of the particles. In peculiar, the derivatization of these gold nanoparticles by aminated biotargeting groups can be envisaged and will constitute our next goal. The specific accumulation of Au@DTDTPA-Gd in tumor is indeed expected to improve the radiotherapy since both the effect of the $\mathrm{X}$-ray dose should be more enhanced by the presence of a higher amount of gold in tumor and healthy tissue should be spared.

\section{Acknowledgement}

This work was supported by the Agence Nationale de la Recherche (ANR-05-NANO-037-02).

\section{About the authors}

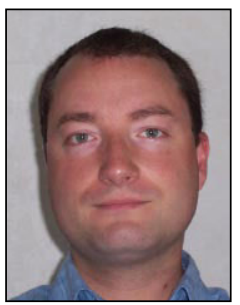

Christophe Alric obtained his Master degree in inorganic chemistry at the University of Lyon (France) and he is now a PhD student in the group of Prof. Olivier Tillement. His research activities are focused on the elaboration of gold nanoparticles combining medical imaging and therapeutic capacities.

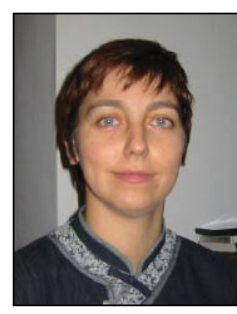

Céline A. Mandon received the Doctorat de spécialité in Biochemistry (2005) from the Université Lyon 1. She was post-doctoral researcher (2005-2006) at the Laboratory of Oxidative Stress, Chaperons and Apoptosis in Lyon (CGMC - CNRS-UMR5534), where she developed bioluminescent and fluorescent stress inducible cell based assays. She is currently post-doc researcher at the laboratory Creatis-LRMN (CNRSUMR-5220), on the Animage platform, where she is in charge of cell labeling by hybrid nanoparticles, used as contrast agents for in vivo imaging.

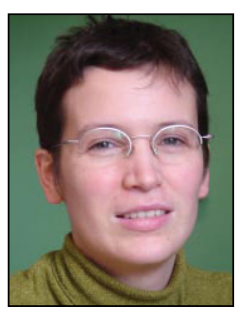

Jacqueline Taleb received a diploma in pharmacy (1998) from the University of Algiers and a Master 2 Research in Biomedical engineering (2007) from the Université Lyon 1. The subject of the training was " $X$-ray radiosensibilizating effects of gold nanoparticules on healthy and tumoral cells". She is currently PhD student at CreatisLRMN (CNRS-UMR-5220), on the Animage platform, where she is developing a tumoral model and testing radiotherapeutic effects of radioactive nanoparticules and their detection by MR, optic and scientigraphic imaging.

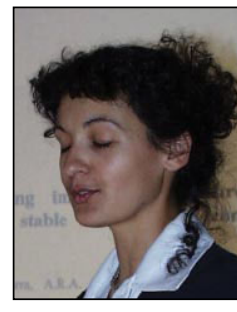

Dr. Géraldine Le Duc obtained her PhD at the interface between biology and physics in 1996. After a one year position in US, she was hired at the ESRF, in order to develop preclinical activities. She is responsible for the BioMedical Facility group providing support and expertise in cellular biology, molecularbiology and in vivo experimentation. She is deeply involved in the ESRF preclinical imaging and radiotherapy program, supervising PhD students and postdoctoral scientists and holding collaborations. 


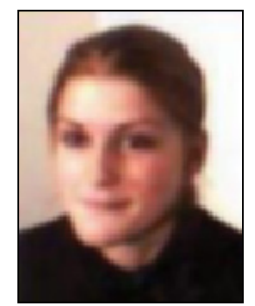

Alice A. Le Meur - Herland received a professional master "imagerie de la santé" (2006) from the Université de Caen BasseNormandie. She did her training period at CYCERON (UMR-CNRS 6185, Caen) where she studied the migration of mesenchymal stem cells at the cerebral level, using a contrast agent, allowing a follow-up by MRI. She is currently engineer on the platform ANIMAGE (Université Claude Bernard Lyon1, CREATIS-LRMN) where she is in charge of in vivo imaging.

Claire Billotey (MD, PhD) is specialized in Medical Biology and Nuclear Medicine. Her professional activities are shared between her medical senior activity in the department of nuclear medicine of the hospital Edouard Herriot (Lyon, France) and her research activities in the University of Lyon 1. Her research interests are focused on the biological evaluation and validation of hybrid nanoparticles designed for biological and medical applications in the field of diagnosis by imaging and therapy.

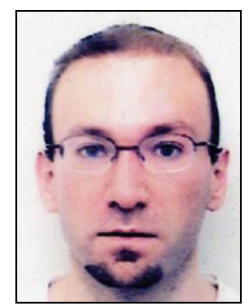

Pascal Perriat 44 years old, is professor at INSA de Lyon and belongs to the laboratory MATEIS (Materials; Science and Engineering). He develops research upon nanoparticles for biology and medicine and is specialized in soft-chemistry routes and characterization at the nanometer scale by Transmission Electron Microscopy. He is the co-author of more than 70 papers and of 5 patents.

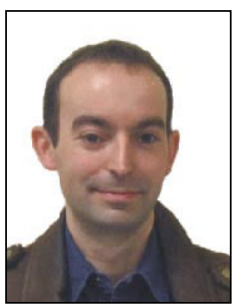

Stéphane Roux graduated in chemistry and electrochemistry from the University of Franche-Comté (France) and completed a PhD degree in Chemistry (conducting hybrid materials) at the same institution in 2000. Afterwards, he moved to the Catholic University of Louvain-la-Neuve (Belgium) for studying the radical polymerization initiated from a solid surface. Since 2002 he is assistant professor at the University Claude Bernard of Lyon (France). His main research activities are focused on the development of nanoparticles combining several imaging techniques and therapy.

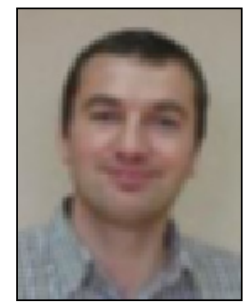

Olivier Tillement studied at the Ecole Normale Supérieure (Paris, France) and obtained his PhD in Chemistry at the university Pierre et Marie Curie in 1992. After a one year position at Tufts University (Boston, USA), he was recruited in 1993 as assistant professor. Olivier Tillement is Professor of Chemistry at the University of Lyon since 2000. The research work of his group includes the synthesis of single crystal fibers for optical applications and of multifunctional nanoparticles for biomedical applications.

\section{References}

1 M.C. Daniel and D. Astruc, Chem. Rev., 2004, 104, 293

2 G. Schmid and B. Corain, Eur. J. Inorg. Chem., 2003, 3081

3 C. Loo, A. Lowery, N.J. Halas, J. West and R. Drezek, Nano Lett., 2005, 5, 709

4 X. Huang, I.H. El-Sayed, W. Qian and M.A. El-Sayed, J. Am. Chem. Soc., 2006, 128, 2115

5 H.S. Choi, W. Liu, P. Misra, E. Tanaka, J.P. Zimmer, B.I. Ipe and M.C. Bawendi, J.V. Frangioni, Nat. Bioltechnol., 2007, 25, 1165

6 J.F. Hainfeld, D.N. Slatkin and H.M. Smilowitz, Phys. Med. Biol., 2004, 49, N309

7 J.F. Hainfeld, D.N. Slatkin, T.M. Focella and H.M. Smilowitz, Brit. J. Radiol., 2006, 79, 248

8 P.-J.Debouttière, S. Roux, F. Vocanson, C. Billotey, O. Beuf, A. Favre-Réguillon, Y. Lin, S. Pellet-Rostaing, R. Lamartine, P. Perriat and O. Tillement, Adv. Funct. Mater., 2006, 16, 2330

9 C. Alric, J. Taleb, G. Le Duc, C. Mandon, C. Billotey, A. Le Meur-Herland, T. Brochard, F. Vocanson, M. Janier, P. Perriat, S. Roux and O. Tillement, J. Am. Chem. Soc., 2008, 130, 5908

10 P. Caravan, J.J. Ellison, T.J. McMurry and R.B. Lauffer, Chem. Rev., 1999, 99, 2293

11 M. Brust, J. Fink, D. Bethell, D.J. Schiffrin and C. Kiely, J. Chem. Soc., Chem. Commun. 1995, 1655

12 J.A. Coderre, T.M. Button, P.L. Micca, C.D. Fisher, M.M. Nawrocky and H.B. Liu, Int. J. Radiat. Oncol. Biol. Phys., 1994, 30, 643

13 P. Benda, K. Someda, J. Messer and W.H. Sweet, J. Neurosurg., 1971, 34, 310

14 N. Kobayashi, N. Allen, N.R. Clendenon and L.W. Ko, J. Neurosurg., 1980, 53, 808

15 G. Paxinos and C. Watson (ed), The Rat Brain in Stereotaxic Coordinates, New York: 1986, Academic

16 H. Elleaume, A.-M. Charvet, G. Berkvens, G. Berruyer, T. Brochard, Y. Dabin, A. Dominguez, A. Draperi, S. Fielder, G. Goujon, G. Le Duc, M. Mattenet, C. Nemoz, M. Perez, M. Renier, C. Schulze,P.Spanne, P.Suortti, W. Thomlinson, F. Esteve, B. Bertrand and J.-F. Le Bas, Nucl. Inst. Meth. Phys. Res. A, 1999, 428, 513

17 J.F. Adam, C. Nemoz, A. Bravin, S. Fielder, S. Bayat, S. Monfraix, G. Berruyer, A. M. Charvet, J.-F. Le Bas, H. Elleaume and J.-F. Estève, J. Cereb. Blood Flow Metab. 2005, 25, 145

18 P. Regnard, G. Le Duc, E. Bräuer-Krisch, I. Troprès, E.A. Siegbahn, A. Kusak, C. Clair, H. Bernard, D. Dallery, J.A. Laissue and A. Bravin, Phys. Med. Biol. 2008, 53, 861

19 J. Folkman, N. Engl. J. Med. 1971, 285, 1182

20 J.P. Duffy, G. Eibl, H.A. Reber and O.J. Hires, Mol. Cancer 2003, 2, 12

21 K. Ichikawa, T. Hikita, N. Maeda, S. Yonezawa, Y. Takeuchi, T. Asai, Y. Namba and N. Oku, Biochim. Biophys. Acta-Biomembr. 2005, 1669, 69 